\title{
Cardiac defects and obesity
}

Despite established links between childhood obesity and adult risk of cardiovascular disease, the independent relationship between childhood obesity and cardiac defects in adulthood is controversial. Now, a new study sheds light on the associations between past and current obesity with adult cardiac structure and function.

The longitudinal study included 159 healthy individuals aged 7-15 years, who were followed up until they were $36-45$ years old. Childhood and adult adiposity was calculated using BMI, and waist circumference measurements were taken at baseline and follow-up. Adiposity was correlated with echocardiographic measurements of cardiac deformation taken at follow-up to determine whether childhood or adult adiposity was associated with adult myocardial dysfunction. "This methodology allowed us to use echo indices as intermediate end points to track the functional disturbances in these 30-40-year-old individuals, which will be a prelude to them developing heart failure (HF)," explains Tom Marwick, an author of the study.

The team reported that childhood adiposity was positively correlated with adult cardiac structural disturbances, including indexed left ventricular mass (LVMi) and left atrial volume (LAVi), but not with adult left ventricular function. Moreover, increased childhood adiposity was associated with a raised risk of increased LVMi and LAVi but not with left ventricular dysfunction, independent of adult adiposity. Conversely, functional myocardial alterations were often associated with high adult adiposity, independent of childhood levels of adiposity.

Overall, the findings demonstrate that childhood adiposity is independently associated with adult structural cardiac disturbances but not with functional defects; the latter are more frequently associated with adult obesity than with childhood adiposity. A follow-up study of this cohort has been funded. "We are hoping that this will give us a stronger picture of what drives the precedents of HF, and therefore HF itself," says Marwick. "If we could identify a target group, early intervention might be possible".

Conor Bradley

ORIGINAL ARTICLE Yang, H. et al. Associations of childhood and adult obesity with left ventricular structure and function. Int. J. Obes. http://dx.doi. org/10.1038/ijo.2016.234 (2017) 